\title{
Axillary Lymph Node Dissection Does Not Improve Post-mastectomy Overall or Disease-Free Survival among Breast Cancer Patients with 1-3 Positive Nodes
}

\author{
Ji Hyeon Joo, MD 1,a \\ Su Ssan Kim, MD, PhD ${ }^{1}$ \\ Byung Ho Son, MD, PhD² \\ Seung Do Ahn, MD, PhD' \\ Jin Hong Jung, MD, $P h D^{1}$ \\ Eun Kyung Choi, MD, PhD' \\ Sei Hyun Ahn, MD, PhD² \\ Jong Won Lee, MD, PhD2 \\ Hee Jeong Kim, MD, PhD² \\ Beom Seok Ko, MD, PhD²
}

Departments of ${ }^{1}$ Radiation Oncology and

${ }^{2}$ Breast and Endocrine Surgery,

Asan Medical Center, University of

Ulsan College of Medicine, Seoul, Korea

Correspondence: Su Ssan Kim, MD, PhD

Department of Radiation Oncology,

Asan Medical Center, University of Ulsan

College of Medicine, 88 Olympic-ro 43-gil,

Songpa-gu, Seoul 05505, Korea

Tel: 82-2-3010-5680

Fax: 82-2-3010-6950

E-mail: watermountain@hanmail.net

Received August 3, 2018

Accepted October 15, 2018

Published Online October 16, 2018

apresent address: Department of Radiation Oncology, Pusan National University Yangsan Hospital, Pusan National University School of Medicine, Yangsan, Korea

\begin{abstract}
Purpose
Axillary lymph node dissection (ALND) may be avoidable for breast cancer patients with 1-2 positive lymph nodes (LN) after breast-conserving therapy. However, the effects of ALND after mastectomy remain unclear because radiation is not routinely used. Herein, we compared the benefits of post-mastectomy ALND versus sentinel node biopsy (SNB) alone for breast cancer patients with 1-3 metastatic LNs.
\end{abstract}

\section{Materials and Methods}

A total of 1,697 patients with pN1 disease who underwent mastectomy during 2000-2015 were identified from an institutional database. Outcomes were compared using the inverse probability of treatment weighted method.

\section{Results}

Patients who underwent SNB tended to have smaller tumors, a lower histology grade, a lower number of positive LNs, and better immunohistochemical findings. After correcting all confounding factors regarding patient, tumor, and adjuvant treatment, the SNB and ALND groups did not differ in terms of overall survival (OS) and disease-free survival (DFS), distant metastasis and locoregional recurrence. The 10 -year DFS and OS rates were $83 \%$ and $84 \%$, respectively, during a median follow-up period of 93 months.

\section{Conclusion}

ALND did not improve post-mastectomy survival outcomes among patients with $\mathrm{N} 1$ breast cancer, even after adjusting for all histopathologic and treatment-related factors.

\section{Key words}

Breast neoplasms, Mastectomy, Sentinel lymph node biopsy, Lymph node excision, Lymph nodes, Disease-free survival

\section{Introduction}

Breast cancer management strategies during the last decade have tended toward less radical surgeries. Accordingly, efforts to limit redundant axillary management have continued. The American College of Surgeons Oncology Group (ACOSOG) Z0011 trial randomized women with up to two positive lymph nodes (LNs) detected after breast-con- serving surgery (BCS) and sentinel node biopsy (SNB) to either the axillary lymph node dissection (ALND) or observation arm [1,2]. In that trial, the regional recurrence rates of $<1 \%$ were equivalent between the arms. However, it remains unclear whether this result is safely applicable to patients after mastectomy. Particularly, radiation is not routinely administered after mastectomy, and therefore post-mastectomy axillary management should different from that after BCS. Currently, about $91 \%$ of women with clinically node- 
negative but SNB-positive LNs underwent completion ALND, while 9\% underwent SNB alone, after mastectomy [3]. Several relevant retrospective analyses have yielded conflicting results [3,4]; while no randomized controlled trials has compared SNB alone vs. ALND, particularly in pN1 stage.

The benefit of post-mastectomy radiotherapy (PMRT) in these patients also remains controversial [5]. According to the 2009 St. Gallen recommendations, PMRT is indicated for patients with $\geq 4$ involved axillary LN [6]; however, indications for its use in patients with 1-3 affected nodes were more restricted and particularly applicable to young patients and those with other poor prognostic features. Accordingly, it remains unclear how these results should be incorporated into clinical practice, given the broad potential for selection bias in breast cancer treatment.

We therefore conducted this study to compare the benefits of ALND vs. SNB alone for breast cancer patients who underwent mastectomy and had 1-3 metastatic LNs. We adjusted for confounding factors to verify our findings. We additionally evaluated the contribution of PMRT to survival outcomes.

\section{Materials and Methods}

\section{Patients}

From 2000 to 2015, 1,768 women with breast cancer underwent total mastectomy and were diagnosed with N1 disease. The patient enrollment algorithm is shown in Fig. 1.

All patients were clinically staged according to the seventh edition of the American Joint Committee on Cancer staging guidelines. Patients were assessed at presentation using the clinical history and findings from the physical examination, mammography, ultrasonography, breast magnetic resonance imaging, and biopsies of the breast and suspicious LNs.

\section{Treatment}

Until 2002, patients were treated with regimens comprising cyclophosphamide, methotrexate, and fluorouracil or cyclophosphamide, doxorubicin, and fluorouracil; since 2003, a taxane-based regimen has been used. Hormone suppression therapy was administered to patients with estrogen receptor-positive or progesterone receptor-positive breast cancer. An anti-human epidermal growth factor receptor 2 (HER-2) targeted agent was administered to patients with a positive HER-2/ neu receptor status.

For pathologic T3 (pT3) tumors, radiotherapy (RT) was administered to the chest wall and regional lymphatics, including the ipsilateral axillary apex and supraclavicular fossa compartment. Radiation was delivered using a 4-15 MV X-ray and/ or 6-16 MeV electron beam from a linear accelerator (Varian Medical Systems, Palo Alto, CA). Total radiation doses of 4,500-5,080 cGy in fractions of 180-250 cGy were typically administered, and boost doses of 540-2,540 cGy were directed to the tumor bed or gross tumor. For T1 $/ 2$ tumors, the use of RT and irradiation field differed in terms of the treating physician's preferences regarding the patient and pathologic characteristics. In this study patients, eight patients with T1/2 tumors received RT after SNB. Radiation field was chest wall and regional lymphatics in four patients, chest wall only in two patients, and unidentified in two patients.

\section{Statistics}

The extent of axillary operation was determined primarily according to the surgical record. However, the number of examined nodes was also considered. Patients were consid-

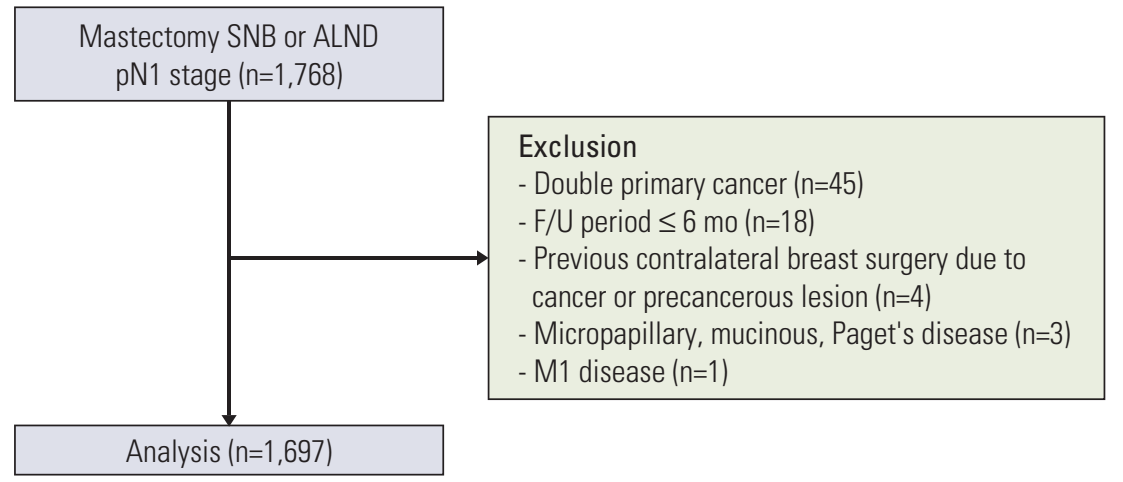

Fig. 1. Patient selection algorithm. SNB, sentinel node biopsy; ALND, axillary lymph node dissection; F/U, follow-up. 
Table 1. Patient characteristics

\begin{tabular}{|c|c|c|c|c|c|c|}
\hline \multirow[b]{2}{*}{ Variable } & \multirow{2}{*}{$\begin{array}{c}\text { Total } \\
(n=1,697)\end{array}$} & \multirow{2}{*}{$\begin{array}{c}\text { ALND } \\
(n=1,539)\end{array}$} & \multirow{2}{*}{$\begin{array}{c}\text { SNB } \\
(n=158)\end{array}$} & \multirow[b]{2}{*}{ p-value } & \multicolumn{2}{|c|}{ SDM } \\
\hline & & & & & $\begin{array}{c}\text { Before } \\
\text { weighting }\end{array}$ & $\begin{array}{c}\text { After } \\
\text { weighting }\end{array}$ \\
\hline Age (yr) & 47.70 & 47.59 & 48.77 & 0.153 & 0.120 & 0.092 \\
\hline BMI $\left(\mathrm{kg} / \mathrm{m}^{2}\right)$ & 23.42 & 23.44 & 23.18 & 0.395 & 0.075 & 0.105 \\
\hline \multicolumn{7}{|l|}{ Histology } \\
\hline IDC & $1,628(95.9)$ & $1,477(96.0)$ & $151(95.57)$ & 0.808 & 0.020 & 0.067 \\
\hline ILC & $69(4.1)$ & $62(4.0)$ & $7(4.43)$ & & & \\
\hline \multicolumn{7}{|l|}{ No. of tumors } \\
\hline 1 & $1,367(80.6)$ & $1,237(80.4)$ & $130(82.3)$ & 0.502 & 0.108 & 0.171 \\
\hline 2 & $243(14.3)$ & $220(14.3)$ & $23(14.6)$ & & & \\
\hline$\geq 3$ & $87(5.1)$ & $82(5.3)$ & $5(3.2)$ & & & \\
\hline \multicolumn{7}{|l|}{ Location $^{\text {a) }}$} \\
\hline UOQ/LOQ & $951(56.5)$ & $861(56.5)$ & $90(57.0)$ & 0.559 & 0.105 & 0.107 \\
\hline Central & $394(23.4)$ & $356(23.3)$ & $38(24.1)$ & & & \\
\hline UIQ/LIQ & $322(19.1)$ & 295 (19.3) & $27(17.1)$ & & & \\
\hline Whole & $16(1.0)$ & $13(0.9)$ & $3(1.9)$ & & & \\
\hline \multicolumn{7}{|l|}{ Histologic grade ${ }^{b)}$} \\
\hline G1 & $61(3.8)$ & $55(3.8)$ & $6(3.9)$ & 0.021 & 0.242 & 0.132 \\
\hline G2 & $928(57.6)$ & $823(56.5)$ & $105(67.7)$ & & & \\
\hline G3 & $622(38.6)$ & $578(39.7)$ & $44(28.4)$ & & & \\
\hline Tumor size (mm) & 28.26 & 28.73 & 23.70 & 0.002 & 0.283 & 0.055 \\
\hline \multicolumn{7}{|l|}{ T category } \\
\hline $\mathrm{T} 1 / 2$ & $1,561(92.0)$ & $1,413(91.8)$ & $148(93.7)$ & 0.413 & 0.072 & 0.064 \\
\hline $\mathrm{T} 3 / 4$ & $136(8.0)$ & $126(8.2)$ & $10(6.3)$ & & & \\
\hline \multicolumn{7}{|l|}{ Positive LN } \\
\hline 1 & 887 (52.3) & 758 (49.3) & $129(81.7)$ & $<0.001$ & 0.772 & 0.175 \\
\hline 2 & $513(30.2)$ & 488 (31.7) & $25(15.8)$ & & & \\
\hline 3 & 297 (17.5) & $293(19.0)$ & $4(2.5)$ & & & \\
\hline \multicolumn{7}{|c|}{ Hormone receptors ${ }^{c}$} \\
\hline Positive & $1,201(71.0)$ & 1,079 (70.3) & $122(78.2)$ & 0.038 & 0.182 & 0.039 \\
\hline Negative & $490(29.0)$ & $456(29.7)$ & $34(21.8)$ & & & \\
\hline \multicolumn{7}{|l|}{ c-Erb B2 ${ }^{d)}$} \\
\hline Negative & 995 (59.3) & $900(59.1)$ & $95(60.9)$ & 0.002 & 0.369 & 0.116 \\
\hline Equivocal & $162(9.7)$ & 159 (10.5) & $3(1.9)$ & & & \\
\hline Positive & $521(31.1)$ & $463(30.4)$ & $58(37.2)$ & & & \\
\hline \multicolumn{7}{|l|}{ Luminal type $^{\mathrm{e})}$} \\
\hline Luminal & $1,201(71.2)$ & 1,079 (70.5) & $122(78.2)$ & 0.042 & & \\
\hline Non-luminal & $486(28.8)$ & $452(29.5)$ & $34(21.8)$ & & & \\
\hline \multicolumn{7}{|l|}{$p 53^{f)}$} \\
\hline $0 / 1+/ 2+$ & $1,249(80.1)$ & $1,131(80.0)$ & $118(80.8)$ & 0.810 & 0.021 & 0.001 \\
\hline $3+$ & 311 (19.9) & $283(20.0)$ & $28(19.2)$ & & & \\
\hline
\end{tabular}

Values are presented as mean or number (\%). ALND, axillary lymph node dissection; SNB, sentinel node biopsy; SDM, standardized difference of means; BMI, body-mass index; IDC, invasive ductal carcinoma; ILC, invasive lobular carcinoma; UOQ, upper outer quadrant; LOQ, lower outer quadrant; UIQ, upper inner quadrant; LIQ, lower inner quadrant; LN, lymph node. ${ }^{\text {a) }} \mathrm{A}$ total of 14 cases had unknown location, ${ }^{\text {b) }} \mathrm{A}$ total of 86 cases had unknown histologic grade, ${ }^{\text {c) }} \mathrm{A}$ total of 6 cases had unknown hormone receptor status, d) A total of 19 cases had unknown c-Erb B2, e) A total of 10 cases had unknown luminal type, ${ }^{\mathrm{f}} \mathrm{A}$ total of 137 cases had unknown p53. 
Table 2. The pattern of first failure sites

\begin{tabular}{lccc} 
& ALND & SNB & $19(7.4)$ \\
Local only & $18(7.3)$ & $1(8.3)$ & $9(3.5)$ \\
Local+regional & $9(3.7)$ & - & $5(2.0)$ \\
Local+distant & $5(2.0)$ & - & $23(9.0)$ \\
Regional only & $20(8.2)$ & $3(25)$ & $42(16.4)$ \\
Regional+distant & $41(16.8)$ & $1(8.3)$ & $142(55.5)$ \\
Distant only & $135(55.3)$ & $7(58.4)$ & $16(6.2)$ \\
Local+regional+distant & $16(6.6)$ & - & $256(100)$ \\
\hline Total & $244(100)$ & $12(100)$ & \\
\hline
\end{tabular}

Values are presented as number (\%). ALND, axillary lymph node dissection; SNB, sentinel node biopsy.
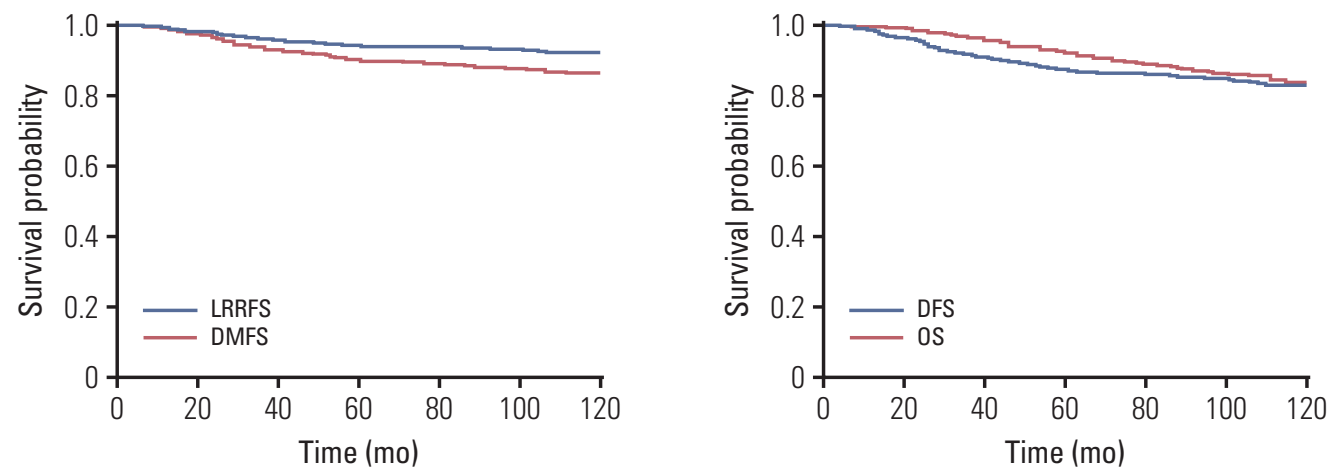

Fig. 2. Kaplan-Meier survival curves of the total patients. LRRFS, lcoregional recurrence-free survival; DMFS, distant metastasis-free survival; DFS, disease-free survival; OS, overall survival.

ered to have undergone an SNB alone if $\leq 5$ nodes were examined. Completed ALND was defined as the examination of $\geq 10$ nodes. This concept was also used in a previous study by the American College of Surgeons [3].

Locoregional failure was defined as recurrent or progressive disease of the ipsilateral chest wall or the regional nodal station (ipsilateral axillary, supraclavicular, or internal mammary LNs). Locoregional failure-free survival (LRFFS), distant metastasis-free survival (DMFS), disease-free survival (DFS), and overall survival (OS) were calculated using the Kaplan-Meier method. Univariate and multivariate analyses were performed using the Cox proportional hazards model. Inter-group comparisons of patient characteristics were performed using the chi-square test. All analyses were 2-tailed, and a $p$-value of $<0.05$ was considered significant. All statistical analyses were performed using the SPSS statistical package, ver. 22.0 (IBM Corp., Armonk, NY).

To adjust for potential selection bias regarding axillary operation, we used an inverse probability of treatment weighted (IPTW) method [7]. The IPTW approach is a propensity score (PS)-based method used to control for confounding factors. This method simulates a sample with balanced characteristics between the two therapy groups, independent of the treatment decision. The PS was derived using a multivariable logistic regression model in which the axillary operation (ALND/SNB alone) was set as the outcome variable. The model included covariates such as the characteristics of the patients and primary tumor, as specified in Table 1 . The $t$ test was used to evaluate continuous variables, and the chi-square test or Fisher exact test was used for categorical variables.

\section{Ethical statement}

Our institutional review board approved the retrospective use of clinical data for this study (2017-1234). As this was a retrospective analysis of routine clinical data, a waiver of the requirement for individual informed consent was granted by the institutional ethics committee. 
Table 3. Univariate and multivariate analyses of LRRFS and DMFS

\begin{tabular}{|c|c|c|c|c|c|c|c|c|}
\hline \multirow{3}{*}{ Variable } & \multicolumn{4}{|c|}{ LRRFS } & \multicolumn{4}{|c|}{ DMFS } \\
\hline & \multicolumn{2}{|c|}{ Univariate } & \multicolumn{2}{|c|}{ Multivariate } & \multicolumn{2}{|c|}{ Univariate } & \multicolumn{2}{|c|}{ Multivariate } \\
\hline & HR & p-value & HR & p-value & HR & p-value & HR & p-value \\
\hline Age & 0.972 & 0.007 & 0.975 & 0.014 & 0.994 & 0.406 & - & - \\
\hline BMI & 0.978 & 0.445 & - & - & 1.050 & 0.012 & 1.046 & 0.020 \\
\hline \multicolumn{9}{|l|}{ No. of tumors } \\
\hline 2 (vs. 1) & 0.716 & 0.276 & - & - & 0.639 & 0.057 & - & - \\
\hline 3 & 1.181 & 0.671 & - & - & 0.707 & 0.337 & - & - \\
\hline \multicolumn{9}{|l|}{ Location } \\
\hline Central (vs. UOQ/LOQ) & 1.331 & 0.228 & 1.298 & 0.270 & 1.279 & 0.154 & 1.270 & 0.162 \\
\hline UIQ/LIQ & 2.051 & 0.001 & 1.850 & 0.006 & 1.495 & 0.023 & 1.502 & 0.022 \\
\hline Whole-breast & 1.567 & 0.656 & 0.673 & 0.702 & 5.450 & $<0.001$ & 2.621 & 0.031 \\
\hline Tumor size & 1.017 & $<0.001$ & 1.015 & 0.002 & 1.019 & $<0.001$ & 1.017 & $<0.001$ \\
\hline Harvested LN & 1.015 & 0.225 & - & - & 1.007 & 0.428 & - & - \\
\hline \multicolumn{9}{|l|}{ Metastatic LN } \\
\hline 2 (vs. 1) & 0.995 & 0.981 & - & - & 1.153 & 0.392 & 1.040 & 0.817 \\
\hline 3 & 1.673 & 0.025 & - & - & 1.876 & $<0.001$ & 1.693 & 0.003 \\
\hline \multicolumn{9}{|l|}{$\mathrm{T}$ category } \\
\hline T $3 / 4$ (vs. T1/2) & 1.498 & 0.185 & - & - & 1.998 & 0.001 & - & - \\
\hline \multicolumn{9}{|l|}{$\mathrm{N}$ category } \\
\hline N1mi (vs. N1) & 0.364 & 0.314 & - & - & 0.417 & 0.219 & - & - \\
\hline \multicolumn{9}{|l|}{ Histologic grade } \\
\hline 2 (vs. 1) & 2.949 & 0.285 & 3.704 & 0.195 & 2.070 & 0.215 & - & - \\
\hline 3 & 6.628 & 0.061 & 5.954 & 0.078 & 3.680 & 0.026 & - & - \\
\hline \multicolumn{9}{|l|}{ Histology } \\
\hline ILC (vs. IDC) & 0.209 & 0.119 & - & - & 0.472 & 0.137 & - & - \\
\hline \multicolumn{9}{|l|}{ Hormone receptor } \\
\hline Negative (vs. positive) & 1.974 & $<0.001$ & - & - & 1.588 & 0.001 & 1.562 & 0.002 \\
\hline \multicolumn{9}{|l|}{ c-Erb B2 } \\
\hline Equivocal (vs. negative) & 1.297 & 0.383 & - & - & 1.644 & 0.017 & - & - \\
\hline Positive & 1.317 & 0.176 & - & - & 1.404 & 0.028 & - & - \\
\hline \multicolumn{9}{|l|}{ Luminal type } \\
\hline Non-luminal (vs. luminal) & 1.988 & $<0.001$ & - & - & 1.600 & 0.001 & - & - \\
\hline \multicolumn{9}{|l|}{ p53 } \\
\hline $3+$ & 1.847 & 0.005 & - & - & 1.392 & 0.063 & - & - \\
\hline \multicolumn{9}{|l|}{ Chemotherapy } \\
\hline Taxane-based & 0.863 & 0.759 & - & - & 0.746 & 0.420 & 0.765 & 0.463 \\
\hline Others & 1.337 & 0.530 & - & - & 1.403 & 0.325 & 1.443 & 0.287 \\
\hline \multicolumn{9}{|l|}{ Radiotherapy } \\
\hline Yes & 1.147 & 0.666 & - & - & 1.912 & 0.001 & - & - \\
\hline \multicolumn{9}{|l|}{ Hormone therapy } \\
\hline Yes & 0.450 & $<0.001$ & 0.522 & 0.002 & 0.696 & 0.015 & - & - \\
\hline \multicolumn{9}{|l|}{ Target agent } \\
\hline Yes & 0.790 & 0.573 & - & - & 0.980 & 0.944 & - & - \\
\hline
\end{tabular}

LRRFS, locoregional recurrence-free survival; DMFS, distant metastasis-free survival; HR, hazard ratio; BMI, body mass index; UOQ, upper outer quadrant; LOQ, lower outer quadrant; UIQ, upper inner quadrant; LIQ, lower inner quadrant; LN, lymph node; ILC, invasive lobular carcinoma; IDC, invasive ductal carcinoma. 


\section{Results}

\section{Patient and tumor characteristics}

A total of 1,697 patients were included in the analysis. The patient and tumor characteristics are shown in Table 1 . The median age was 47.7 years (range, 23 to 81 years). The majority of patients $(95.9 \%)$ had ductal carcinoma and underwent $\operatorname{ALND}(91 \%)$.

\section{Patterns of the first failure}

The patterns of the first failure are summarized in Table 2. Most of the 256 patients who experienced recurrence had distant metastases (205/256). Isolated local and regional recurrences were detected in 19 and 23 patients, respectively. The most frequent sites of distant metastasis were the bone and lung. In 34 patients, the first recurrence involved metastases at $>3$ sites. Regional recurrences of the axillary, supraclavicular, and internal mammary LNs were diagnosed in 41, 47, and 36 patients, respectively. The sites of recurrence are specified in S1 Table.

\section{Prognostic factors for survival}

The median follow-up period was 93 months (range, 3 to 212 months). The 10-year LRRFS, DMFS, DFS, and OS rates were $92 \%, 86 \%, 83 \%$, and $84 \%$, respectively (Fig. 2). The results of a prognostic factor analysis of LRRFS and DMFS are shown in Table 3. In the multivariate analysis, age, location, tumor size, and hormone therapy were identified as significant factors for LRRFS, whereas the body mass index, location, size, number of positive LNs, and hormone receptor status were significant factors for DMFS. The analyses of DFS and OS are summarized in Table 4. The tumor location, size, number of metastatic LNs, and hormone receptor status were identified as poor prognostic factors for DFS and OS. Additionally, the histologic grade was predictive of DFS while the body mass index and chemotherapy were predictive of OS.

\section{Inverse probability of treatment weighted}

To account for the effects of confounding factors, we calculated a PS and used this value in an IPTW analysis. Based on the prognostic factor analysis, our covariates included age, body mass index, number of breast tumors (1 vs. 2 vs. $\geq 3$ ), tumor location (outer quadrant vs. inner quadrant vs. central vs. whole breast), size, number of metastatic LNs (1 vs. 2 vs. 3), T category (T1/2 vs. T3/4), histologic grade (1 vs. 2 vs. 3), histologic type (invasive ductal carcinoma vs. invasive lobular carcinoma), hormone receptor status (posi- tive vs. negative), HER-2/neu receptor status (positive vs. negative), luminal type (luminal vs. non-luminal), and p53 expression (negative/weak/intermediate vs. strong). We additionally adjusted for the following known postoperative prognostic factors: number of harvested LNs, N category (N1 vs. N1mi), use of adjuvant chemotherapy, adjuvant RT, adjuvant hormone therapy, and trastuzumab therapy. A numeric distribution of patients is shown in S2 Table. A median of 15.2 LNs were retrieved. Fifty-two patients (3\%) were diagnosed with N1mi stage disease.

The hazard ratios of locoregional recurrence, distant metastasis, progression, and death for the SNB group relative to the ALND group are shown in Table 5. After IPTW correction and additional adjustments of postoperative covariates, we observed no differences in LRRFS, DFS, and OS between the SNB and ALND groups. Rather, only the risk of distant metastasis was significantly lower in the SNB group after IPTW correction. Specifically, the hazard ratio for distant metastasis was 0.35 (95\% confidence interval, 0.12 to 0.97; $\mathrm{p}=0.043$ ). This difference was lost after additional adjustments of postoperative covariates. The results are shown in Fig. 3.

\section{Discussion}

In this study, we reviewed patients who had undergone mastectomy at our institution and were determined to have pN1 or pN1mi disease. After a median follow-up of 93 months, we observed no significant differences in the LRRFS, DMFS, DFS, and OS rates of patients who underwent SNB vs. those who underwent ALND. We observed a clear bias toward smaller tumors, lower histologic grade, lower number of metastatic LNs, and better immunohistochemical findings among patients who underwent a limited axillary operation. To correct this, we applied the IPTW method. Subsequently, we observed no differences in OS, DFS, and LRRFS between the groups. The corrected DMFS rate was higher in the SNB group relative to the ALND group, however, after adjusting for postoperative factors, the statistical power was lost.

Despite ongoing controversy, the avoidance of axillary node dissection in selected patients with micrometastatic disease or isolated tumor cells in the sentinel node is considered adequate $[3,8]$. However, the issue of whether to complete ALND after a notification of nodal macrometastases remains controversial. The results of the ACOSOG Z0011 trial suggest that ALND can be avoided safely in patients with up to 2 macroscopically positive sentinel nodes [2]. The long-term follow-up also showed excellent regional control, despite the 
Table 4. Univariate and multivariate analyses of DFS and OS

\begin{tabular}{|c|c|c|c|c|c|c|c|c|}
\hline \multirow{3}{*}{ Variable } & \multicolumn{4}{|c|}{ DFS } & \multicolumn{4}{|c|}{ OS } \\
\hline & \multicolumn{2}{|c|}{ Univariate } & \multicolumn{2}{|c|}{ Multivariate } & \multicolumn{2}{|c|}{ Univariate } & \multicolumn{2}{|c|}{ Multivariate } \\
\hline & HR & p-value & HR & p-value & HR & p-value & HR & p-value \\
\hline Age & 0.988 & 0.080 & - & - & 1.001 & 0.849 & - & - \\
\hline BMI & 1.030 & 0.103 & - & - & 1.054 & 0.004 & 1.050 & 0.007 \\
\hline \multicolumn{9}{|l|}{ No. of tumors } \\
\hline 2 (vs. 1) & 0.583 & 0.014 & - & - & 0.554 & 0.014 & - & - \\
\hline 3 & 0.933 & 0.809 & - & - & 1.026 & 0.929 & - & - \\
\hline \multicolumn{9}{|l|}{ Location } \\
\hline Central (vs. UOQ/LOQ) & 1.219 & 0.202 & 1.216 & 0.206 & 1.316 & 0.083 & 1.343 & 0.061 \\
\hline UIQ/LIQ & 1.498 & 0.010 & 1.505 & 0.009 & 1.382 & 0.057 & 1.442 & 0.032 \\
\hline Whole-breast & 4.984 & $<0.001$ & 2.269 & 0.046 & 8.403 & $<0.001$ & 4.072 & $<0.001$ \\
\hline Tumor size & 1.018 & $<0.001$ & 1.015 & $<0.001$ & 1.018 & $<0.001$ & 1.012 & 0.000 \\
\hline Harvested LN & 1.009 & 0.271 & - & - & 1.006 & 0.471 & - & - \\
\hline \multicolumn{9}{|l|}{ Metastatic LN } \\
\hline 2 (vs. 1) & 1.211 & 0.193 & 1.121 & 0.445 & 1.252 & 0.146 & 1.144 & 0.393 \\
\hline 3 & 1.824 & - & - & 0.002 & 2.029 & $<0.001$ & 1.898 & $<0.001$ \\
\hline \multicolumn{9}{|l|}{$\mathrm{T}$ category } \\
\hline T $3 / 4$ (vs. T1/2) & 1.794 & 0.002 & - & - & 2.240 & $<0.001$ & - & - \\
\hline \multicolumn{9}{|l|}{$\mathrm{N}$ category } \\
\hline N1mi (vs. N1) & 0.481 & 0.208 & - & - & 0.131 & 0.152 & - & - \\
\hline \multicolumn{9}{|l|}{ Histologic grade } \\
\hline 2 (vs. 1) & 2.568 & 0.107 & 2.249 & 0.112 & 1.690 & 0.305 & 1.602 & 0.306 \\
\hline 3 & 4.694 & 0.008 & 3.119 & 0.027 & 3.347 & 0.017 & 2.266 & 0.078 \\
\hline \multicolumn{9}{|l|}{ Histology } \\
\hline ILC (vs. IDC) & 0.369 & 0.048 & - & - & 0.561 & 0.201 & - & - \\
\hline \multicolumn{9}{|l|}{ Hormone receptor } \\
\hline Negative (vs. positive) & 1.713 & $<0.001$ & 1.443 & 0.010 & 1.998 & $<0.001$ & 1.612 & 0.001 \\
\hline \multicolumn{9}{|l|}{ c-Erb B2 } \\
\hline Equivocal (vs. negative) & 1.624 & 0.010 & - & - & 1.594 & 0.016 & - & - \\
\hline Positive & 1.365 & 0.024 & - & - & 1.560 & 0.002 & - & - \\
\hline \multicolumn{9}{|l|}{ Luminal type } \\
\hline Non-luminal (vs. luminal) & 1.726 & $<0.001$ & - & - & 1.995 & $<0.001$ & - & - \\
\hline \multicolumn{9}{|l|}{ p53 } \\
\hline Strong & 1.570 & 0.003 & - & - & 1.643 & 0.002 & - & - \\
\hline \multicolumn{9}{|l|}{ Chemotherapy } \\
\hline Taxane-based & 0.597 & 0.082 & 0.617 & 0.105 & 0.272 & $<0.001$ & 0.287 & $<0.001$ \\
\hline Others & 1.108 & 0.712 & 1.159 & 0.597 & 0.539 & 0.007 & 0.562 & 0.012 \\
\hline \multicolumn{9}{|l|}{ Radiotherapy } \\
\hline Yes & 1.685 & 0.005 & - & - & 2.108 & $<0.001$ & - & - \\
\hline \multicolumn{9}{|l|}{ Hormone therapy } \\
\hline Yes & 0.611 & $<0.001$ & - & - & 0.531 & $<0.001$ & - & - \\
\hline \multicolumn{9}{|l|}{ Target agent } \\
\hline Yes & 0.896 & 0.680 & - & - & 0.673 & 0.201 & - & - \\
\hline
\end{tabular}

DFS, disease-free survival; OS, overall survival; HR, hazard ratio; BMI, body mass index; UOQ, upper outer quadrant; LOQ, lower outer quadrant; UIQ, upper inner quadrant; LIQ, lower inner quadrant; LN, lymph node; ILC, invasive lobular carcinoma; IDC, invasive ductal carcinoma. 
Table 5. Outcome analysis based on the IPTW method

\begin{tabular}{|c|c|c|c|}
\hline Outcome & $\mathrm{HR}^{\mathrm{a})}$ & $95 \% \mathrm{CI}$ & p-value \\
\hline \multicolumn{4}{|l|}{ Overall survival (event=237/1,697) } \\
\hline Univariate & 0.457 & $0.225-0.926$ & 0.030 \\
\hline Multivariable adjusted & 0.675 & $0.330-1.380$ & 0.281 \\
\hline IPTW method ${ }^{\text {b) }}$ & 0.537 & $0.212-1.356$ & 0.188 \\
\hline IPTW and adjusted by covariatesc) & 0.566 & $0.219-1.464$ & 0.240 \\
\hline \multicolumn{4}{|l|}{ Locoregional recurrence-free survival } \\
\hline Univariate & 0.499 & $0.203-1.224$ & 0.129 \\
\hline Multivariable adjusted & 0.624 & $0.254-1.537$ & 0.306 \\
\hline IPTW method ${ }^{\text {b) }}$ & 0.698 & $0.248-1.967$ & 0.497 \\
\hline IPTW and adjusted by covariates $\left.{ }^{c}\right)$ & 0.775 & $0.251-2.394$ & 0.658 \\
\hline \multicolumn{4}{|l|}{ Distant metastasis-free survival } \\
\hline Univariate & 0.444 & 0.219-0.901 & 0.025 \\
\hline Multivariable adjusted & 0.622 & $0.304-1.275$ & 0.195 \\
\hline IPTW method ${ }^{\text {b) }}$ & 0.346 & 0.124-0.965 & 0.043 \\
\hline IPTW and adjusted by covariates ${ }^{\mathrm{c}}$ & 0.374 & $0.131-1.063$ & 0.065 \\
\hline \multicolumn{4}{|l|}{ Disease-free survival } \\
\hline Univariate & 0.530 & $0.297-0.947$ & 0.032 \\
\hline Multivariable adjusted & 0.752 & $0.417-1.357$ & 0.344 \\
\hline IPTW method ${ }^{\text {b) }}$ & 0.552 & $0.254-1.200$ & 0.134 \\
\hline IPTW and adjusted by covariates ${ }^{c}$ & 0.619 & $0.327-1.172$ & 0.141 \\
\hline
\end{tabular}

$\mathrm{CI}$, confidence interval. a) Hazard ratio (HR): comparison of the sentinel node biopsy group vs. the axillary node dissection group, ${ }^{b}$ Inverse-probability-of-treatment weighted (IPTW) method, ${ }^{c}$ Adjusted for postoperatively determined covariates (harvested lymph nodes, $\mathrm{N}$ category, radiotherapy, hormone therapy, and target agents).

potential for residual axillary disease [9]. Additionally, ALND could be avoided in a large majority of Z0011-eligible patients, regardless of the use of routine nodal radiation [10]. However, the applicability of these findings to patients undergoing total mastectomy has not been established. Several publications from single-institutional studies have reported the outcomes of mastectomy cases. The majority of these studies suggest that omitting ALND or replacing it with RT does not worsen DFS $[4,11]$. Accordingly, the pattern of axillary management has shifted toward less radical surgery (Fig. 4) $[3,4,8,10]$.

Nodal radiation is another alternative to ALND for LNpositive patients. In the current analysis, adjuvant RT did not improve LRRFS, DFS, or OS. In this study, we sought to identify a patient group that would benefit from RT. However, the retrospective nature of this study precluded such an analysis because most of the high-risk patients underwent ALND. Still, other researchers have attempted such an analysis. In the 2005 pooled analysis of the Early Breast Cancer Trialists' Collaborative Group (EBCTCG), PMRT yielded reductions in the risks of local recurrence and 15 -year breast cancer mortality [12]. As RT led to similar proportional reductions in the rate of local recurrence among all women, large absolute reductions in local recurrence were only observed if the control risk was also large. Therefore, PMRT was indicated for N2 disease; however, the benefit of PMRT in N1 disease, which is assumed to confer a relatively low risk of absolute locoregional recurrence, remained debatable. Recently, a meta-analysis showed that a locoregional recurrence reduction in response to PMRT conferred a significant survival benefit upon patients with N1 disease [13]. Chang et al. [5] performed a retrospective study of patients who underwent mastectomy plus ALND [5]; in this group, 17.8\% of patients also received PMRT. Here, PMRT did not significantly reduce the locoregional recurrence ( $1 \%$ vs. $3.8 \%, 5$ years) but was associated with an improved DFS. As shown above, the evidence supports the use of PMRT for patients with $\mathrm{N} 1$ disease who did not undergo ALND. In contrast to cases involving BCS, for which adjuvant radiation is scheduled, post-mastectomy patients face a more complicated decision-making process regarding RT. The current American Society of Clinical Oncology (ASCO) guidelines recommend that "Clinicians should not recommend ALND for women with early-stage breast cancer who have one or two sentinel lymph node metastases and will receive breast-conserving surgery with conventionally fractionated whole-breast $R T^{\prime \prime}$; this recommendation is based on the ACOSOG Z0011 and International Breast Cancer Study Group (IBCSG)-2301 clinical trials [2,14]. This 


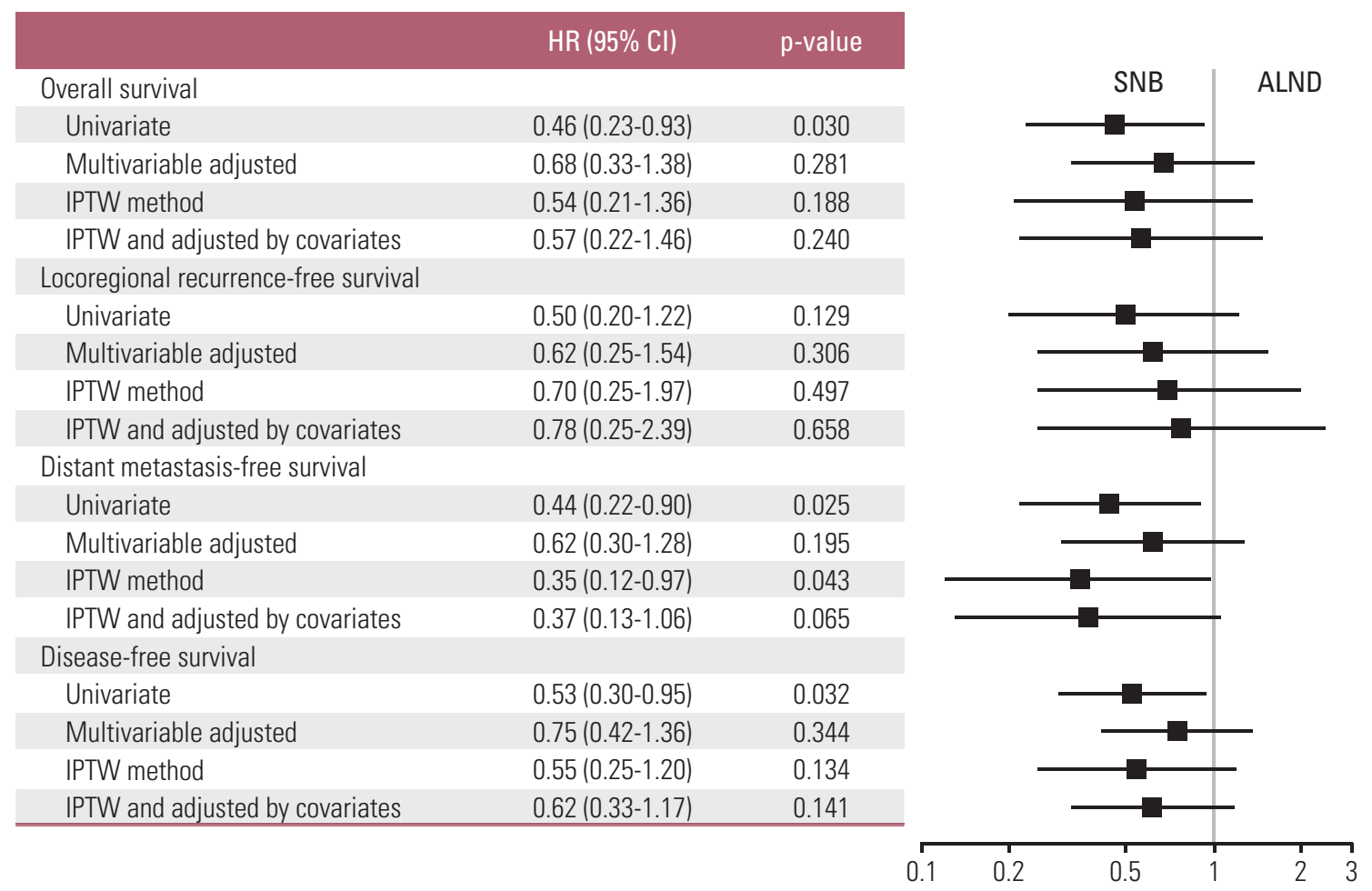

Fig. 3. Forest plot demonstrating the risks of death, locoregional recurrence, distant metastasis, and disease recurrences for sentinel node biopsy (SNB) relative to axillary lymph node dissection (ALND). HR, hazard ratio; CI, confidence interval; IPTW, inverse probability of treatment weighted.

suggests that no definitive guidelines on axillary management exist for cases in which RT is not planned, such as mastectomy cases involving T1 or T2 breast cancer.

Previous research has identified age, tumor size, premenopausal status, and the numbers of positive and dissected LNs as predictors of locoregional failure in patients with node-positive breast cancer [15]. Recent studies that included patients treated with modern chemotherapy regimens reported that the presence of extensive intraductal components, lymphovascular invasion, histologic grade 3, and non-luminal subtype were also predictive of local control $[16,17]$. A current study revealed similar findings. Additionally, patients with inner quadrant tumors tended to had unfavorable outcomes, including LRRFS, DMFS, DFS, and OS. According to several studies conducted with respect to tumor location as a prognostic factor, inner quadrant tumors were associated with a lower OS rate and more frequent distant metastases, compared to outer quadrant tumors [18]. Although it remains unclear why patients with inner quadrant breast cancer have worse outcomes, a potential association with internal mammary node (IMN) involvement is generally accepted. More aggressive chemotherapy or PMRT that includes the IMN area can be considered in patients with

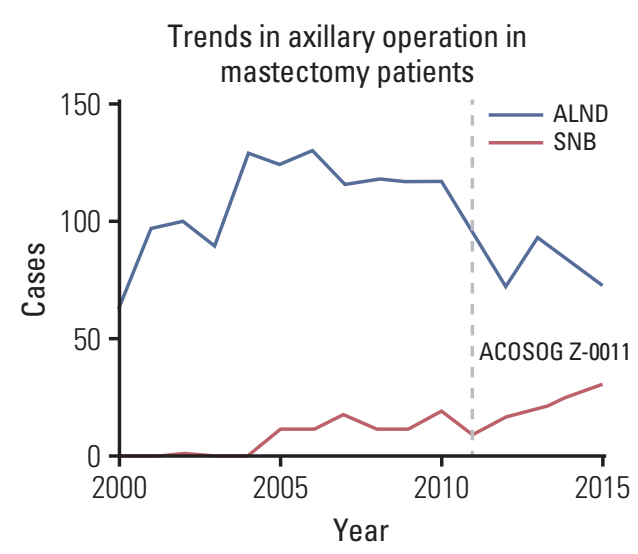

Fig. 4. Changes in the number of axillary lymph node dissection (ALND) and sentinel node biopsy (SNB) procedures by year.

inner quadrant tumors. In this study, which includes a large number of patients with $\mathrm{N} 1$ disease, at least three metastatic LNs was also identified as a poor prognosticator for DMFS, DFS, and OS. Metastatic LNs may be a source of early 
regional or distant recurrence; therefore, the extent of LN metastasis can serve as a prognosticator in breast cancer patients with metastatic LNs. A population-based study of more than 25,000 women found that the LN ratio (LNR, the ratio of positive LNs to the total number of removed LNs) was an important prognostic factor independent of traditional clinicopathological factors [19]. Some researchers have argued that the LNR may have a greater prognostic value than the absolute number of involved nodes. Moreover, the LNR has been identified as a significant predictor of outcomes in all stages of breast cancer [20]. Given the variability of factors related to the local recurrence of breast cancer, it is difficult to determine the need for PMRT using only ALND. Further research is needed to define the appropriate indications for PMRT. We suggest that less aggressive axillary procedures for N1 patients should be considered cautiously for those with inner quadrant tumors or metastasis to $\geq 3 \mathrm{LNs}$. Additionally, age, tumor size, and hormone receptor status should be considered when making decisions about adjuvant treatment.

Our study had several limitations of note, including those inherent to a retrospective analysis. First, the long study period led to heterogeneity in the use of chemotherapy regimens. Second, patients were not randomly allocated to undergo ALND vs. SNB alone or RT vs. no RT. We used
IPTW-PS matching to adjust for these errors. However, it was difficult to draw any definitive conclusions regarding the efficacy of ALND or PMRT. A randomized clinical trial of this subject is currently ongoing in the Netherlands. Specifically, the Dutch Breast Cancer Research Group (BOOG) 201307 trial is conducting a randomized clinical trial to determine whether completion axillary treatment can be safely omitted in SNB-positive breast cancer patients treated with mastectomy. Until the results of that study are published, our findings could serve as a reference for treatment decisions.

In conclusion, ALND did not improved the survival outcomes, including locoregional control, of women with pN1 breast cancer who underwent mastectomy, even after adjusting for all histopathologic and treatment-related factors. Omitting ALND or replacing it with RT in these patient groups can be considered in the absence of high risk factors.

\section{Electronic Supplementary Material}

Supplementary materials are available at Cancer Research and Treatment website (https:// www.e-crt.org).

\section{Conflicts of Interest}

Conflict of interest relevant to this article was not reported.

\section{References}

1. Giuliano AE, McCall L, Beitsch P, Whitworth PW, Blumencranz P, Leitch AM, et al. Locoregional recurrence after sentinel lymph node dissection with or without axillary dissection in patients with sentinel lymph node metastases: the American College of Surgeons Oncology Group Z0011 randomized trial. Ann Surg. 2010;252:426-32.

2. Giuliano AE, Hunt KK, Ballman KV, Beitsch PD, Whitworth PW, Blumencranz PW, et al. Axillary dissection vs no axillary dissection in women with invasive breast cancer and sentinel node metastasis: a randomized clinical trial. JAMA. 2011;305: 569-75.

3. Bilimoria KY, Bentrem DJ, Hansen NM, Bethke KP, Rademaker AW, Ko CY, et al. Comparison of sentinel lymph node biopsy alone and completion axillary lymph node dissection for node-positive breast cancer. J Clin Oncol. 2009;27:2946-53.

4. Fu Y, Chung D, Cao MA, Apple S, Chang H. Is axillary lymph node dissection necessary after sentinel lymph node biopsy in patients with mastectomy and pathological N1 breast cancer? Ann Surg Oncol. 2014;21:4109-23.

5. Chang JS, Lee J, Kim KH, Sohn JH, Kim SI, Park BW, et al. Do recent advances in diagnostic and therapeutic procedures negate the benefit of postmastectomy radiotherapy in N1 pati- ents with a low risk of locoregional recurrence? Medicine (Baltimore). 2015;94:e1259.

6. Goldhirsch A, Ingle JN, Gelber RD, Coates AS, Thurlimann B, Senn HJ, et al. Thresholds for therapies: highlights of the St Gallen International Expert Consensus on the primary therapy of early breast cancer 2009. Ann Oncol. 2009;20:1319-29.

7. Austin PC, Stuart EA. Moving towards best practice when using inverse probability of treatment weighting (IPTW) using the propensity score to estimate causal treatment effects in observational studies. Stat Med. 2015;34:3661-79.

8. Yi M, Giordano SH, Meric-Bernstam F, Mittendorf EA, Kuerer HM, Hwang RF, et al. Trends in and outcomes from sentinel lymph node biopsy (SLNB) alone vs. SLNB with axillary lymph node dissection for node-positive breast cancer patients: experience from the SEER database. Ann Surg Oncol. 2010;17 Suppl 3:343-51.

9. Giuliano AE, Ballman K, McCall L, Beitsch P, Whitworth PW, Blumencranz $\mathrm{P}$, et al. Locoregional recurrence after sentinel lymph node dissection with or without axillary dissection in patients with sentinel lymph node metastases: long-term follow-up from the American College of Surgeons Oncology Group (Alliance) ACOSOG Z0011 randomized trial. Ann Surg. 
2016;264:413-20.

10. Morrow M, Van Zee KJ, Patil S, Petruolo O, Mamtani A, Barrio $\mathrm{AV}$, et al. Axillary Dissection and nodal irradiation can be avoided for most node-positive Z0011-eligible breast cancers: a prospective validation study of 793 patients. Ann Surg. 2017;266:457-62.

11. Milgrom S, Cody H, Tan L, Morrow M, Pesce C, Setton J, et al. Characteristics and outcomes of sentinel node-positive breast cancer patients after total mastectomy without axillary-specific treatment. Ann Surg Oncol. 2012;19:3762-70.

12. Clarke M, Collins R, Darby S, Davies C, Elphinstone P, Evans $\mathrm{V}$, et al. Effects of radiotherapy and of differences in the extent of surgery for early breast cancer on local recurrence and 15-year survival: an overview of the randomised trials. Lancet. 2005;366:2087-106.

13. EBCTCG (Early Breast Cancer Trialists' Collaborative Group), McGale P, Taylor C, Correa C, Cutter D, Duane F, et al. Effect of radiotherapy after mastectomy and axillary surgery on 10-year recurrence and 20-year breast cancer mortality: metaanalysis of individual patient data for 8135 women in 22 randomised trials. Lancet. 2014;383:2127-35.

14. Galimberti V, Cole BF, Zurrida S, Viale G, Luini A, Veronesi $\mathrm{P}$, et al. Axillary dissection versus no axillary dissection in patients with sentinel-node micrometastases (IBCSG 23-01): a phase 3 randomised controlled trial. Lancet Oncol. 2013;14: 297-305.
15. Taghian A, Jeong JH, Mamounas E, Anderson S, Bryant J, Deutsch $\mathrm{M}$, et al. Patterns of locoregional failure in patients with operable breast cancer treated by mastectomy and adjuvant chemotherapy with or without tamoxifen and without radiotherapy: results from five National Surgical Adjuvant Breast and Bowel Project randomized clinical trials. J Clin Oncol. 2004;22:4247-54.

16. Lai SF, Chen YH, Kuo WH, Lien HC, Wang MY, Lu YS, et al. Locoregional recurrence risk for postmastectomy breast cancer patients with T1-2 and one to three positive lymph nodes receiving modern systemic treatment without radiotherapy. Ann Surg Oncol. 2016;23:3860-9.

17. Yu JI, Park W, Choi DH, Huh SJ, Nam SJ, Kim SW, et al. Prognostic modeling in pathologic N1 breast cancer without elective nodal irradiation after current standard systemic management. Clin Breast Cancer. 2015;15:e197-204.

18. Lohrisch C, Jackson J, Jones A, Mates D, Olivotto IA. Relationship between tumor location and relapse in 6,781 women with early invasive breast cancer. J Clin Oncol. 2000;18:2828-35.

19. Dings PJ, Elferink MA, Strobbe LJ, de Wilt JH. The prognostic value of lymph node ratio in node-positive breast cancer: a Dutch nationwide population-based study. Ann Surg Oncol. 2013;20:2607-14.

20. Woodward WA, Vinh-Hung V, Ueno NT, Cheng YC, Royce $\mathrm{M}$, Tai $\mathrm{P}$, et al. Prognostic value of nodal ratios in node-positive breast cancer. J Clin Oncol. 2006;24:2910-6. 